\title{
PBT2 Rapidly Improves Cognition in Alzheimer's Disease: Additional Phase II Analyses
}

\author{
Noel G. Faux ${ }^{\mathrm{a}}$, Craig W. Ritchie ${ }^{\mathrm{b}}$, Adam Gunn ${ }^{\mathrm{a}}$, Alan Rembach ${ }^{\mathrm{a}, \mathrm{c}}$, Andrew Tsatsanis ${ }^{\mathrm{a}}$, Justin Bedo ${ }^{\mathrm{d}}$, \\ John Harrison ${ }^{\mathrm{e}}$, Lars Lannfelt ${ }^{\mathrm{f}}$, Kaj Blennow ${ }^{\mathrm{g}}$, Henrik Zetterberg ${ }^{\mathrm{g}}$, Martin Ingelsson ${ }^{\mathrm{f}}$, \\ Colin L. Masters ${ }^{\mathrm{a}}$, Rudolph E. Tanzi ${ }^{\mathrm{h}}$, Jeffrey L. Cummings ${ }^{\mathrm{i}}$, Caroline M. Herd ${ }^{\mathrm{j}}$ and Ashley I. Bush ${ }^{\mathrm{a}, \mathrm{k}, *}$ \\ ${ }^{a}$ Mental Health Research Institute, The University of Melbourne, Parkville, VIC, Australia \\ ${ }^{\mathrm{b}}$ Department of Psychological Medicine, Imperial College London, London, UK \\ ${ }^{\mathrm{c}}$ CSIRO Parkville, VIC, Australia \\ ${ }^{\mathrm{d}}$ Victorian Research Laboratory, National ICT of Australia (NICTA) \\ ${ }^{\text {e }}$ CogState Ltd, Warminster, UK \\ ${ }^{\mathrm{f}}$ Department of Public Health / Geriatrics, Uppsala University Hospital, Uppsala, Sweden \\ ${ }^{g}$ Clinical Neurochemistry Laboratory, Sahlgren's University Hospital, Mölndal, Sweden \\ ${ }^{\mathrm{h}}$ Genetics and Aging Research Unit, Massachusetts General Hospital, Boston, MA, USA \\ ${ }^{\mathrm{i}}$ Mary S. Easton Center for Alzheimer's Disease Research at UCLA, Los Angeles, CA, USA \\ ${ }^{j}$ Prana Biotechnology Ltd, Parkville, Victoria, Australia \\ ${ }^{\mathrm{k}}$ Department of Pathology, The University of Melbourne, Parkville, VIC, Australia
}

\begin{abstract}
PBT2 is a copper/zinc ionophore that rapidly restores cognition in mouse models of Alzheimer's disease (AD). A recent Phase IIa double-blind, randomized, placebo-controlled trial found that the $250 \mathrm{mg}$ dose of PBT2 was well-tolerated, significantly lowered cerebrospinal fluid (CSF) levels of amyloid- $\beta_{42}$, and significantly improved executive function on a Neuro-psychological Test Battery (NTB) within 12 weeks of treatment in patients with AD. In the post-hoc analysis reported here, the cognitive, blood marker, and CSF neurochemistry outcomes from the trial were subjected to further analysis. Ranking the responses to treatment after 12 weeks with placebo, PBT2 $50 \mathrm{mg}$, and PBT2 $250 \mathrm{mg}$ revealed that the proportions of patients showing improvement on NTB Composite or Executive Factor z-scores were significantly greater in the PBT2 $250 \mathrm{mg}$ group than in the placebo group. Receiver-operator characteristic analyses revealed that the probability of an improver at any level coming from the PBT2 $250 \mathrm{mg}$ group was significantly greater, compared to placebo, for Composite z-scores (Area Under the Curve $[$ AUC] $=0.76, p=0.0007$ ), Executive Factor z-scores (AUC $=0.93, p=1.3 \times 10^{-9}$ ), and near-significant for the ADAS-cog (AUC $=0.72, p=0.056$ ). There were no correlations between changes in CSF amyloid- $\beta$ or tau species and cognitive changes. These findings further encourage larger-scale testing of PBT2 for AD.
\end{abstract}

Keywords: Alzheimer's disease, clinical trials Randomized controlled, cognition, PBT2

Supplement available online: http://www.j-alz.com/issues/20/vol20-2.html\#supplementarydata

\footnotetext{
${ }^{*}$ Correspondence to: Professor Ashley I. Bush, The Mental Health Research Institute, 155 Oak Street, Parkville, VIC 3052, Australia.
}

Tel.: +61 39389 2962; Fax: +61 39387 5061; E-mail: a.bush@ mhri.edu.au. 


\section{INTRODUCTION}

PBT2 is potentially a disease-modifying drug for Alzheimer's disease (AD). As a zinc/copper ionophore, PBT2 has been shown in animal models to facilitate the clearance of amyloid- $\beta(\mathrm{A} \beta)$ aggregates in the cortex by targeting the zinc and copper ions that mediate the assembly of these aggregates in amyloid and diffuse deposits, effectively detoxifying the $\mathrm{A} \beta$. Copper and zinc ions are released during glutamatergic neurotransmission and glial activation and promote the formation of $\mathrm{A} \beta$ oligomers and amyloid deposits [1]. The ionophoric properties of PBT2 liberate copper and zinc ions trapped by amyloid, facilitating the reuptake of these essential metal ions into cells which is believed to promote memory functions such as long-term potentiation $[2,3]$. Oral PBT2 treatment is strikingly effective in amyloid- $\beta$ protein precursor transgenic mouse models for $\mathrm{AD}$, markedly improving learning and memory within days, accompanied by a reduction in interstitial $\mathrm{A} \beta[2]$.

PBT2 was tested at $50 \mathrm{mg}$ and $250 \mathrm{mg}$ daily oral doses in patients with early $\mathrm{AD}$ in a double-blind, placebo-controlled Phase IIa randomized clinical trial over 12 weeks of treatment. The drug was welltolerated, induced a $12 \%$ drop in cerebrospinal fluid (CSF) A $\beta_{42}$ levels, and produced a significant improvement in Executive Factor z-score on a Neuropsychological Test Battery (NTB) [4].

Here, we report the results of a post-unblinding analysis of the cognitive data that was not included in the original paper. We re-analyzed the data to rank the members of the treatment cohorts for cognitive benefits of PBT2, and also determined whether there were associations with biochemical outcomes.

\section{MATERIALS AND METHODS}

\section{Cohort and trial description}

The cohort and the treatment protocol have been previously reported [4]. Briefly, this was a 12-week, double blind, randomized, parallel group, placebocontrolled trial of two doses of PBT2 $(50 \mathrm{mg}$ and $250 \mathrm{mg}$ oral capsules daily). The study was approved by the Medicines Control Agency and the central ethics committees (Sweden) or the human research ethics committees at each center, with notification to the Therapeutic Goods Administration (Australia). Written informed consent was obtained from all patients. All patients met
NINCDS-ADRDA criteria for probable AD [5], had early AD (Mini-Mental Status Examination [MMSE] Score 20-26, and/or Alzheimer's Disease Assessment Scale-cognitive subscale [ADAS-cog] score 10-25), and had been taking an acetylcholinesterase inhibitor for at least 4 months before entry. Primary outcomes were safety and tolerability. Key secondary outcomes were plasma and CSF biomarkers (including CSF tau, phospho-tau Thr181 [pTau], $\mathrm{A} \beta_{40}$, and $\mathrm{A} \beta_{42}$ ) and cognition (NTB, ADAS-cog, and MMSE). 78 patients were recruited and randomized between December 6, 2006 and December 28, 2007 at 8 sites in Sweden and 7 sites in Australia. The study was registered at ClinicalTrials.gov, number NCT00471211.

\section{Statistical analysis}

All statistical analysis was performed using $\mathrm{R}$ version 2.90 [6]. Linear regression was performed to investigate the correlation between cognitive changes, from pretreatment to week 12, and concomitant changes CSF levels of $\mathrm{A} \beta_{40}, \mathrm{~A} \beta_{42}$, total tau (tTau), and pTau. Tests of the biochemical changes were corrected for multiple testing by controlling the false discovery rate using the Benjamin-Hochberg method [7] to generate adjusted p-values.

Receiver-operator characteristic (ROC) curves were analyzed according to previously published protocols for adapting clinical trial data [8]. In this approach, True positives are scores from subjects who improved while on PBT2 $250 \mathrm{mg}$, while False positives are subjects who improved while on placebo.

\section{NTB Composite z-scores}

The NTB composite z-score is composed of the following tests: Wechsler Memory Scale (WMS) Verbal Paired Associates test (immediate and delayed), Rey Auditory Verbal Learning Test (RAVLT) test (immediate and delayed), WMS Digit Span, Category Fluency Test, Controlled Oral Word Association Test (COWAT), and Trail Making Test parts A and B. The Memory Factor z-score is composed of the following tests: WMS Verbal Paired associates test (immediate and delayed) and RAVLT test (immediate and delayed). The Executive Factor z-score is composed of the following tests: WMS Digit Span, COWAT, Category Fluency test, and Trail Making Test parts A and B.

\section{$A \beta$ and tau}

$\mathrm{A} \beta_{40}, \mathrm{~A} \beta_{42}$, tau, and pTau levels in CSF were assayed by ELISA as previously described [4]. 


\section{Plasma oxidase activity}

The assay was based upon that of Schosinsky and colleagues [9]. Plasma samples were incubated at $37^{\circ} \mathrm{C}$ after being mixed with $75 \mathrm{mM}$ sodium acetate buffer $\mathrm{pH}$ 5.0. $7.88 \mathrm{mM}$ o-dianisidine dihydrochloride substrate (Sigma) was added, mixed and then incubated. Sulfuric acid ( $9 \mathrm{M}$ ) was added to stop the reaction at 5 and $60 \mathrm{~min}$. Oxidized product was monitored by absorbance at $540 \mathrm{~nm}$.

\section{Western blot}

Samples were separated by polyacrylamide gel electrophoresis (Invitrogen) under non-reducing, denaturing conditions and transferred to a nitrocellulose membrane. Non-specific binding was blocked with 5\% fatfree dry milk in TBS-T. The membrane was washed and incubated $\left(0.5 \mu \mathrm{g} / \mathrm{mL}, 4^{\circ} \mathrm{C}\right.$, overnight $)$ with polyclonal anti-ceruloplasmin (Dako), or mouse monoclonal anti-SOD1 (Ab20926, Abcam). The washed membrane was then incubated with peroxidase-conjugated anti-rabbit or anti-mouse immunoglobulin (1:10,000, Dako, $1 \mathrm{~h}, \mathrm{RT})$. Immunoreactive proteins were visualized by chemiluminescence (ECL, Amersham), captured (LAS-3000 Imaging suite) and analyzed (Multi Gauge, Fuji).

\section{SOD1 analysis}

Erythrocytes extracts were prepared as described [10], with minor modification. Briefly, haemolysates were prepared by adding washed erythrocytes (200 $\mu \mathrm{L})$ to $\mathrm{ddH}_{2} \mathrm{O}(1400 \mu \mathrm{L})$ and incubating on ice for $10 \mathrm{~min}$. Aliquots $(250 \mu \mathrm{L})$ of haemolysate were added to an equal volume of ice-cold ethanol, vortexed (5 s), then ice-cold chloroform added $(100 \mu \mathrm{L})$, and vortexed again $(30 \mathrm{~s})$. The mixtures were centrifuged $(3000 \mathrm{~g}$, $10 \mathrm{~min}, 4^{\circ} \mathrm{C}$ ), and the upper (aqueous) phase removed and stored at $-80^{\circ} \mathrm{C}$ until used. For SOD1 activity, extracts were assayed using a microtiter-plate kit (SOD Assay Kit Cat\# S311, Dojindo Molecular Technologies). For SOD1 quantitation, neat extracts were added to sample buffer (NuPAGE LDS, Invitrogen) containing TCEP $(50 \mathrm{mM})$, and analyzed by Western Blotting, compared to standards of purified human erythrocyte SOD1 (Cat\# S9636, Sigma).

\section{Plasma free copper assay}

The presence of exchangeable or free copper ions in plasma was measured by detection of copperphenanthroline-dependent DNA degradation with minor modifications. To a $1.5 \mathrm{~mL}$ tube the following were added in order: thymus DNA (80 $\mu \mathrm{L}$, $2 \mathrm{mg} / \mathrm{ml}), 1,10$-phenanthroline $(20 \mu \mathrm{L}, 1 \mathrm{mM}), \mathrm{NaN}_{3}$ $(10 \mu \mathrm{L}, 100 \mathrm{mM}), \mathrm{CuCl}_{2}$ or neat plasma $(10 \mu \mathrm{L})$, phosphate buffer $(40 \mu \mathrm{L}, 0.25 \mathrm{M}, \mathrm{pH} 6.4)$, and $2-\beta$ mercaptoethanol $(15 \mu \mathrm{L}, 0.4 \%, \mathrm{v} / \mathrm{v})$. The samples were then incubated for $1 \mathrm{~h}$ at $37^{\circ} \mathrm{C}$. EDTA $(20 \mu \mathrm{L}, 0.1 \mathrm{M})$ was then added to stop the reaction, followed by thiobarbituric acid $(100 \mu \mathrm{L} 1 \% \mathrm{w} / \mathrm{v}$ in $50 \mathrm{mM} \mathrm{NaOH})$ and trichloroacetic acid $(100 \mu \mathrm{L}, 28 \% \mathrm{w} / \mathrm{v})$, and boiling for $10 \mathrm{~min}$. An additional blank was prepared for each sample containing water instead of the 1,10phenanthroline. A standard curve of $\mathrm{CuCl}_{2}$ concentrations (1 to $10 \mu \mathrm{M}$ ) was prepared. After cooling, each sample was extracted into butan-1-ol $(600 \mu \mathrm{L})$. Phases were separated by centrifugation (2000 g, $5 \mathrm{~min}$ ), and the fluorescence of the butan-1-ol phase measured (em $553 \mathrm{~nm}$, ex 532nm).

\section{RESULTS}

\section{Distribution of NTB changes}

The least squares (ls) mean differences provide only a rudimentary description of treatment effects. We therefore analyzed the NTB z-score changes in greater depth by ranking the ls mean change from pretreatment at 12 weeks for each member of the treatment cohorts, as has been used previously to support the efficacy of donepezil for AD in early Phase II studies [11]. Most subjects showed improved performance at 12 weeks on each of the z-scores (Fig. 1), indicating a considerable learning effect even in this cohort of patients with mild dementia. The greatest learning effect, as exhibited by placebo results, was for the Memory Factor z-scores, where $100 \%$ of the placebo subjects performed better than pre-treatment (Fig. 1B). 93\% of the placebo subjects scored better for the Executive Factor and Composite z-scores than at pre-treatment. Importantly for the calculation of the Composite z-scores, the dimension of the learning effect in the placebo group was approximately 3-times greater for the Memory Factor z-scores than for the Executive Factor Z-scores (Fig. 1).

Despite the learning effects in the placebo group, the percentage of patients who improved was greater 
A

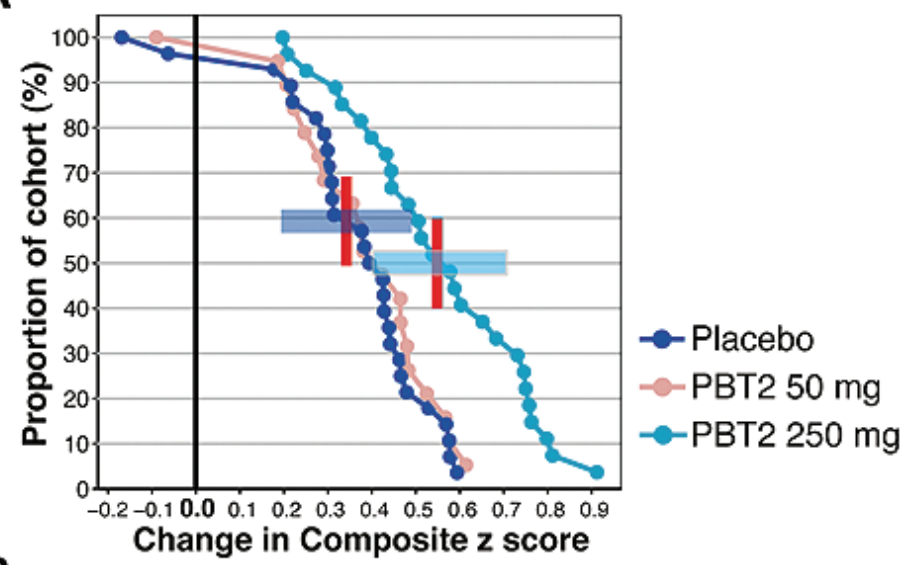

B

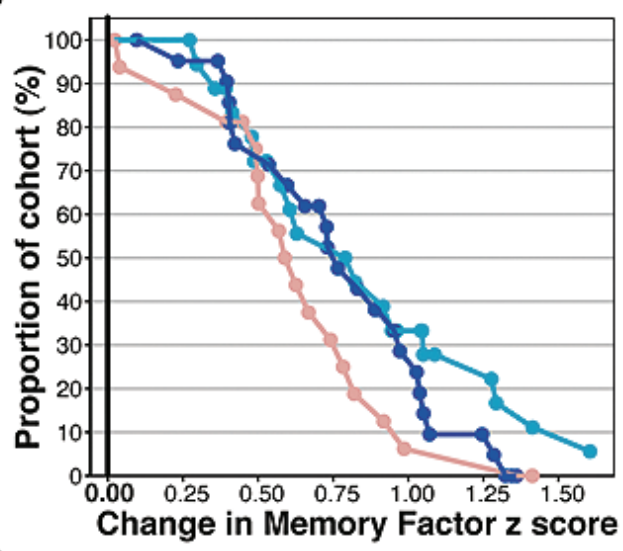

C

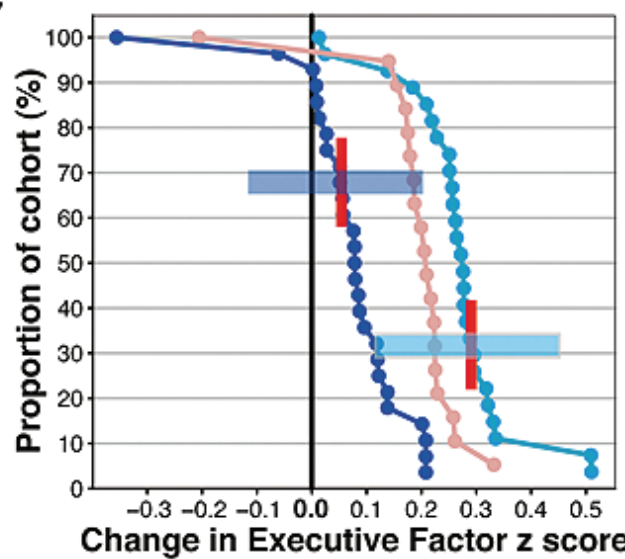

Fig. 1. Response ranking of treatment groups. Plot of the cumulative percentage of patients within each of the three treatment groups who showed an ls-means difference equal to or greater than a given value shown on the $\mathrm{x}$ axis. The red vertical lines show the means for the group where a significant difference is noted in later analysis. The shaded areas are the 95\% confidence intervals. A) Ranking of NTB Composite z-scores, B) Ranking of NTB Memory Factor z-scores, C) Ranking of NTB Executive Factor z-scores. For the NTB tests a positive change from pretreatment is an improvement.

in the PBT2 250 mg group than the placebo group for all thresholds of improvement in the Composite and Executive Factor z-scores (Fig. 1A and C). $41 \%$ of the
PBT2 $250 \mathrm{mg}$ cohort responded better on the Composite z-score than then best response in the placebo group (Fig. 1A). 81\% of the PBT2 $250 \mathrm{mg}$ cohort responded 
Table 1

Correlations of CSF biomarker readouts. The $\mathrm{r}^{2}$ and $\mathrm{p}$-value (in parenthesis) of the linear regression analyses of changes in CSF biomarkers for the whole cohort from pre-treatment to week 12 are shown. Those results in bold face showed a significant or near-significant linear relationship between two CSF biochemical markers

\begin{tabular}{lccc}
\hline & $\Delta \mathrm{A} \beta_{40}$ & $\Delta$ Total Tau & $\Delta$ Phospho Tau \\
\hline$\Delta \mathrm{A} \beta_{42}$ & $\mathbf{0 . 4 4 4}\left(\mathbf{2 . 2 6} \times \mathbf{1 0}^{-10}\right)$ & $0.000(0.996)$ & $0.018(0.335)$ \\
$\Delta \mathrm{A} \beta_{40}$ & & $0.021(0.335)$ & $\mathbf{0 . 0 7 1}(\mathbf{0 . 0 5 2})$ \\
$\Delta$ Total Tau & & & $\mathbf{0 . 0 8 4}(\mathbf{0 . 0 4 5})$ \\
\hline
\end{tabular}

better on the Executive Factor Z-score than the best response in the placebo group (Fig. 1C). The best 35\% of Memory Factor z-score responses for PBT2 $250 \mathrm{mg}$ group were uniformly higher than the best $35 \%$ of the placebo group (Fig. 1B), indicating that the drug was inducing a greater proportion of larger improvements in memory performance than placebo.

\section{ROC analysis of the likelihood that a cognitive improver was on PBT2 $250 \mathrm{mg}$}

To apply a statistical analysis to the distribution of improvements induced by PBT2 $250 \mathrm{mg}$ treatment, we performed a ROC analysis adapted to clinical trials [8]. This test analyzed whether treatment with PBT2 $250 \mathrm{mg}$ was more likely than placebo to induce an improvement of any dimension in the performance readouts. In this analysis, all the 12-week responses (differences from pretreatment) for a given z-score test were ranked, and then the probability of the response coming from the PBT2 $250 \mathrm{mg}$ treatment group (True positive) or the placebo group (False positive) was calculated. This revealed that improvement of any dimension on the NTB Composite z-score was significantly more likely when on PBT2 than on placebo (AUC = $0.76, p=0.0007$, Fig. 2A). The curve also revealed that the greatest likelihood of differences between the PBT2 treatment group and the placebo group were in the range of a z-score of $0.49-0.71$ above the pretreatment performance score (Fig. 2A). The ROC for the NTB Executive Factor $\mathrm{z}$-scores revealed that improvement of any dimension was also more likely in the PBT2 treatment group than in the placebo group (AUC $=$ $0.93, p=1.3 \times 10^{-9}$ ), with the greatest likelihood of differences between the PBT2 treatment group and the placebo group at a z-score of 0.2 above the pretreatment performance score (Fig. 2C). The ROC of the Memory Factor z-scores found no significant effects of either PBT2 or placebo: there was a trend for improvement in the z-score range of $0.99-1.31$, the larger end of the response rank, to be more likely in the PBT2-treated group (Fig. 2B).
Because the ROC analysis appeared to allow us to discriminate better than ls means differences between the PBT2 $250 \mathrm{mg}$ and placebo group, we also analyzed the MMSE and ADAS-cog data. MMSE showed no significant difference (not shown) but for ADAS-cog the AUC was 0.72 in favor of PBT2 $250 \mathrm{mg}$, which was a trend to improvement ( $p=0.056$, Fig. 2D).

\section{Effects of PBT2 on blood metalloenzymes and "free" copper}

PBT2 and its predecessor clioquinol are sometimes misunderstood to represent pharmacological chelators, although preclinical evidence indicates that these compounds act as ionophores $[2,13]$, and a Phase IIa clinical trial of clioquinol found that plasma zinc levels rose with treatment and copper levels were unaffected [12]. PBT2 treatment in the current trial did not alter total plasma copper or zinc levels [4]. To test whether the drug impacted on major metalloproteins or free copper levels, which have been implicated in AD [13], we assayed ceruloplasmin and copper/zinc superoxide dismutase specific activities in plasma and red cells respectively, as well as plasma "free" (exchangeable) copper levels. These measurements were part of the Statistical Analysis Plan, but were not previously reported. No changes were seen in the placebo or PBT2 treatment groups over the 12 weeks of the study (Supplementary Tables 1 and 2), indicating that PBT2 does not perturb peripheral metal metabolism.

\section{Correlations of CSF $A \beta$ and tau changes}

We examined the relationship of the CSF biomarkers (A $\beta_{40}, \mathrm{~A} \beta_{42}$, tTau, and pTau) to each other and to the cognitive outcomes. We found that a change in $\mathrm{A} \beta_{40}$ significantly correlated with a change in $\mathrm{A} \beta_{42}$, a change in tTau significantly correlated with a change in pTau, and a change in $\mathrm{A} \beta_{40}$ near-significantly correlated with a change in pTau (Table 2). These associations were significant only when the combined data from the treatment and placebo cohorts were pooled. There were no significant association of changes in these biomarkers with changes in cognitive outcomes. 

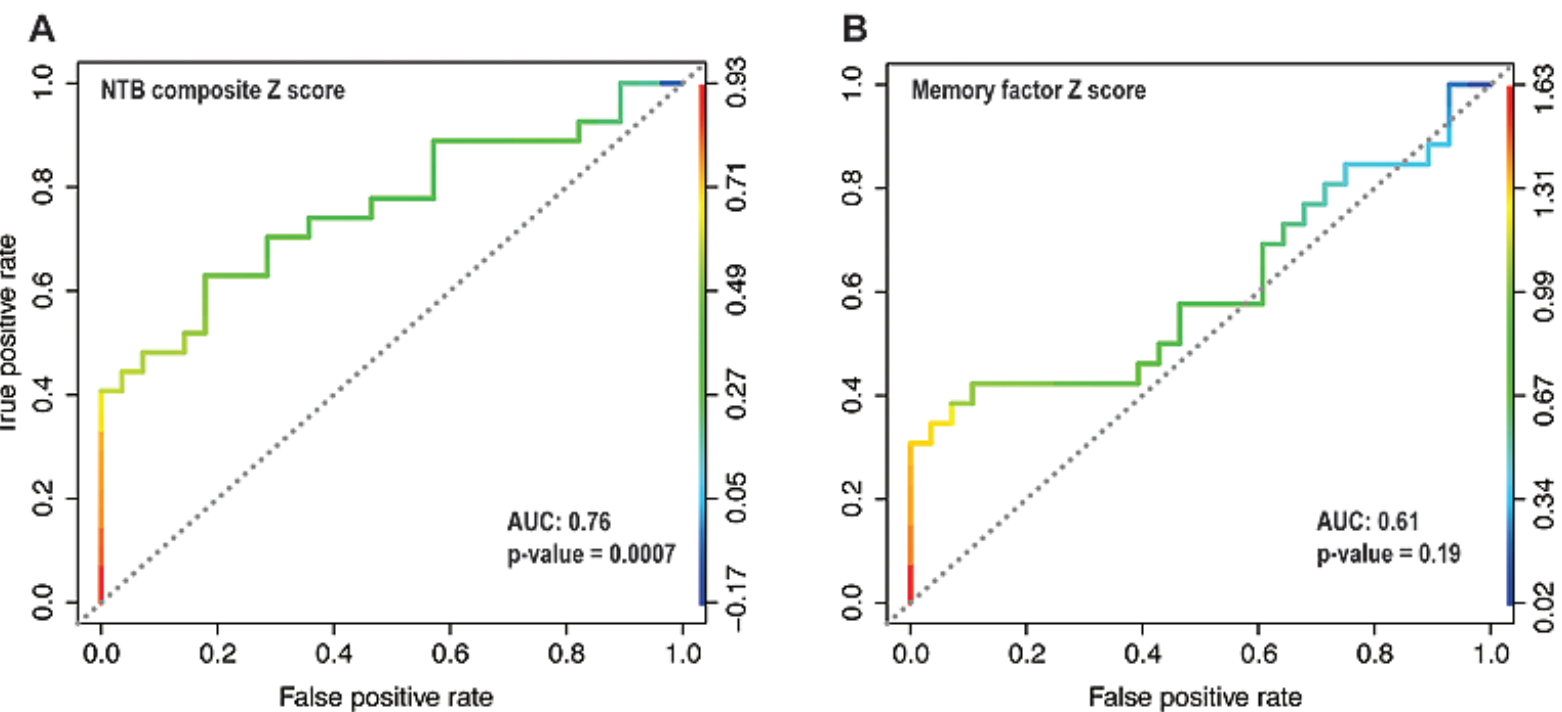

C

D
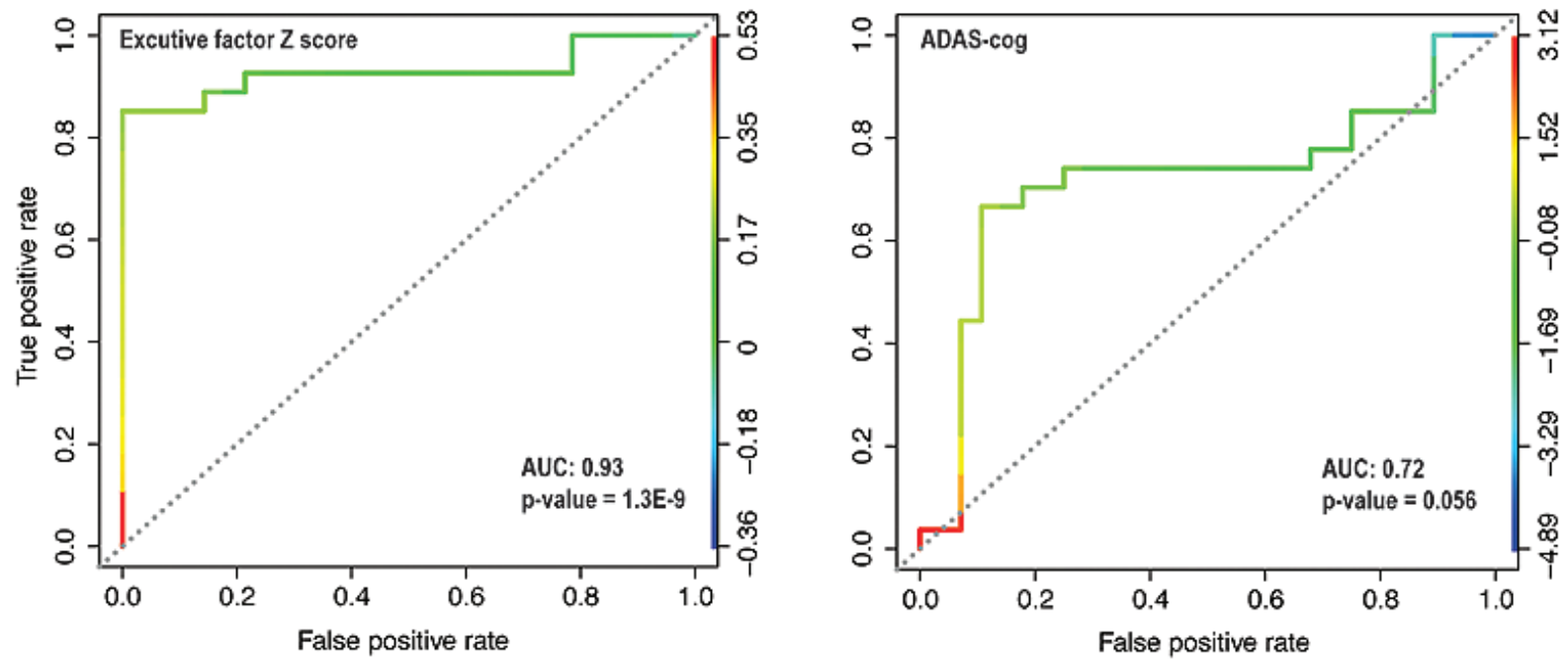

Fig. 2. Receiver-operator characteristics (ROC) curves between ranked placebo and PBT2 $250 \mathrm{mg}$ responses. All ls-means difference changes of NTB z-scores between pre-treatment to 12 weeks for the placebo and PBT2 $250 \mathrm{mg}$ cohorts were ranked and tested for whether a given threshold of response fell in the placebo or PBT2 group. The left $y$-axis and the x-axis express the probability of a participant with a given 1s-means difference being in the PBT2 $250 \mathrm{mg}$ group or placebo group. The right y-axis and color code indicate the threshold of improvement corresponding to the associated point on the ROC. A) ROC curve for the NTB Composite z-scores. B) ROC curve for the NTB Memory Factor z-scores. C) ROC curve for the NTB Executive Factor z-scores. D) ROC curve for the ADAS-cog scores. AUC, area under the curve. The p-value indicates a significant change from 0.5 , the dashed line, which indicates random association $(\mathrm{AUC}=0.5)$. For NTB tests a positive value is improvement while for ADAS-cog a negative value is improvement.

\section{Biochemical responders analysis}

For the analyses reported here we defined five "biochemical responders" groups, based upon contemporary thinking about the potential of $\mathrm{CSF} \mathrm{A} \beta$ and tau as predictive markers for AD progression [14], as well as our previous observations that PBT2 lowers interstitial brain $\mathrm{A} \beta_{42}$ levels in transgenic mice [2]. A decrease in $\mathrm{A} \beta_{40}$ and $\mathrm{A} \beta_{42}$, a decrease in tTau and pTau, a de- crease in all of $\mathrm{A} \beta_{40}, \mathrm{~A} \beta_{42}$, tTau, and pTau, an increase in the ratio of $\mathrm{A} \beta_{40} / \mathrm{A} \beta_{42}$, and a decrease in the ratio of $\mathrm{A} \beta_{42} / \mathrm{p}$ Tau were analyzed. We then tested to determine if there was any difference between the placebo and PBT2 $250 \mathrm{mg}$ group within and between the defined biochemical responder groups for NTB Composite z-scores, Executive Factor z-scores and Memory Factor z-scores. After correction for multiple testing there were no significant differences within and be- 
tween the biochemical improvers, placebo and PBT2 $250 \mathrm{mg}$ group (data not shown).

\section{DISCUSSION}

A disease-modifying treatment for $\mathrm{AD}$ has proven elusive. Approaches that showed promise in preclinical testing were not authenticated in randomized Phase II and III clinical trials [15-17]. The primary outcomes of our Phase IIa clinical trial were safety and tolerability, with cognitive improvement as a secondary outcome. While there is great interest in determining whether cognitive improvement is evident even in early-phase AD clinical trials, this is especially difficult with a disease-modifying approach, where little cognitive deterioration is expected in short time frames in a placebo group; in our case, 12 weeks. Our initial report of PBT2 treatment over 12 weeks in patients with early AD described that the drug was safe and well-tolerated at $50 \mathrm{mg}$ and $250 \mathrm{mg}$ doses, and that the $250 \mathrm{mg}$ dose induced a significant decrease in CSF $\mathrm{A} \beta_{42}$, and a significant improvement in the NTB Executive Factor $\mathrm{z}$ score [4]. A cognitive battery similar to the NTB has been shown to reliably assess cognitive changes in patients with mild AD [18,19], and may be more sensitive to change in patients with mild AD or early disease, compared to ADAS-cog and MMSE, which are more suited to measurement of patients with more advanced disease. We found the NTB to be more discriminatory than ADAS-cog and MMSE in discerning treatmentassociated cognitive improvement in this study.

The ranking analysis reported here has been previously used to demonstrate the efficacy of donepezil for AD in early Phase II studies [11]. Using this approach there was a striking separation, with no overlap, between PBT2 $250 \mathrm{mg}$ and placebo cohorts in the ranked responses to treatment for Composite z-scores and Executive Factor Z-scores (Fig. 1A and C). This effect could not be appreciated from the primary efficacy analysis that compared the overlapping standard errors of the ls means [4]. To test whether PBT2 $250 \mathrm{mg}$ had a significant benefit in this ranked analysis, we utilized ROC curve statistics, which were originally devised to discriminate ambiguous data, and recently validated as an analysis for Phase 2 clinical trials [8]. The ROC analysis confirmed that the treatment effects of PBT2 $250 \mathrm{mg}$ compared to placebo over 12 weeks were significant: $p=0.0007$ for NTB Composite z-score, largely driven by the Executive Factor component ( $p=$ $1.3 \times 10^{-9}$, Fig. 2 C), but also driven by the best $35 \%$ of responders on the Memory Factor Z-scores (Figs 2B, $3 \mathrm{~B})$. ROC analysis also revealed a trend $(p=0.056)$ towards a benefit of PBT2 $250 \mathrm{mg}$ treatment over placebo on ADAS-cog (Fig. 2D). Therefore the ranking and ROC analyses provided more confidence in the validity of a PBT2-mediated treatment benefit than the ls means differences for these readouts. Efficacy signals in early Phase II clinical trials are prone to Type II error because of uncertainties about optimal dose and treatment duration, and because it is premature to utilize large numbers of patients. The ranking and ROC analyses may therefore be useful approaches to avoid Type II error at this early phase.

Effects on Executive Function (EF) may prove to be of particular utility in helping to understand drug efficacy in AD clinical studies. Recent data indicate that patients with mild AD, treated with donepezil, showed greater effect-size improvements on the NTB as compared with the ADAS-cog [9]. The same study also found a correlation $\left(\mathrm{r}^{2}=0.6\right)$ between performance on the NTB and the Disability Assessment for Dementia (DAD). These findings suggest that the NTB, which contains well-known measures of EF, can capture important positive effects on cognition that are less readily detected by traditional measures. The correlation between the NTB and DAD suggests that the inclusion of EF measures improves the ability to predict overall function from cognitive abilities.

We conclude that PBT2 $250 \mathrm{mg}$ treatment for 12 weeks was highly likely to have induced more cognitive improvement than placebo in this mildly affected $\mathrm{AD}$ cohort. In $\mathrm{AD}$ animal models, marked cognitive improvement was seen within days of commencing treatment with PBT2 despite the drug lacking neurotransmitter-mediated effects [2]. We hypothesize that improvement was due to rapid clearance of oligomeric $\mathrm{A} \beta$ from the brain [2]. Our observations in animals and patients indicate that PBT2's potential disease-modifying effect may be observable early, impacting upon a modifiable disease component where reversible neurotoxicity mediates symptoms, in contrast to later stage irreversible neurodegeneration. As a disease-modifying approach, the effect size of clinical benefit is expected to increase with duration of treatment, which encourages the study of PBT2 in larger scale clinical trials of longer duration.

Another feature of our study was an attempt to determine whether changes from baseline in CSF A $\beta$ or tau species could serve as biomarkers for cognitive change. We found no significant correlative associations, despite significantly decreased CSF $\mathrm{A} \beta_{42}$ levels and sig- 
nificant EF z-score improvement in the PBT treatment group compared to placebo. This study is probably underpowered to discern such correlations, but could help in the design of future studies.

\section{ACKNOWLEDGMENTS} Ltd.

This study was sponsored by Prana Biotechnology

Drs. Tanzi, Masters, Ritchie, and Bush are consultants for and shareholders in Prana Biotechnology Ltd. Drs. Harrison and Cummings are paid consultants to Prana Biotechnology Ltd. Dr. Herd is an employee of, and shareholder in, Prana Biotechnology Ltd. Dr Harrison is an employee of Cogstate Ltd. Dr. Faux is a National Health and Medical Research Council (NHMRC) training fellow.

Dr. Tanzi has no input into the drafting or review of any portion of the manuscript other than those portions pertaining to study concept and design and consultation on biochemistry, and no input into the analysis of the data from other portions.

Authors' disclosures available online (http://www.jalz.com/disclosures/view.php?id=281).

\section{REFERENCES}

[1] Bush AI (2008) Drug development based on the metals hypothesis of Alzheimer's disease. J Alzheimers Dis 15, 223240.

[2] Adlard PA, Cherny RA, Finkelstein DI, Gautier E, Robb E, Cortes M, Volitakis I, Liu X, Smith JP, Perez K, Laughton K, Li QX, Charman SA, Nicolazzo JA, Wilkins S, Deleva K, Lynch T, Kok G, Ritchie CW, Tanzi RE, Cappai R, Masters CL, Barnham KJ, Bush AI (2008) Rapid restoration of cognition in Alzheimer's transgenic mice with 8-hydroxy quinoline analogs is associated with decreased interstitial Abeta. Neuron 59, 43-55.

[3] Bush AI, Tanzi RE (2008) Therapeutics for Alzheimer's disease based on the metal hypothesis. Neurotherapeutics 5, 421432.

[4] Lannfelt L, Blennow K, Zetterberg H, Batsman S, Ames D, Harrison J, Masters CL, Targum S, Bush AI, Murdoch R, Wilson J, Ritchie CW (2008) Safety, efficacy, and biomarker findings of PBT2 in targeting Abeta as a modifying therapy for Alzheimer's disease: a phase IIa, double-blind, randomised, placebo-controlled trial. Lancet Neurol 7, 779-786.

[5] McKhann G, Drachman D, Folstein MF, Katzman R, Price D, Stadlen E (1984) Clinical diagnosis of Alzheimer's disease: Report of the NINCDS-ADRDA work group under the auspices of the Department of Health and Human Services Task Force on Alzheimer's Disease. Neurology 34, 939-944.
[6] RDevelopmentCoreTeam (2009) R: A Language and Environment for Statistical Computing.

[7] Benjamini Y, Hochberg Y (1995) Controlling the false discovery rate: a practical and powerful approach to multiple testing. $J$ R Stat Soc Series B Stat Methodol 57, 289-300.

[8] Brumback LC, Pepe MS, Alonzo TA (2006) Using the ROC curve for gauging treatment effect in clinical trials. Stat Med 25, 575-590.

[9] Schosinsky KH, Lehmann HP, Beeler MF (1974) Measurement of ceruloplasmin from its oxidase activity in serum by use of o-dianisidine dihydrochloride. Clin Chem 20, 1556-1563.

[10] Peskin AV, Winterbourn CC (2000) A microtiter plate assay for superoxide dismutase using a water-soluble tetrazolium salt (WST-1). Clin Chim Acta 293, 157-166.

[11] Rogers SL, Farlow MR, Doody RS, Mohs R, Friedhoff LT (1998) A 24-week, double-blind, placebo-controlled trial of donepezil in patients with Alzheimer's disease. Donepezil Study Group. Neurology 50, 136-145.

[12] Ritchie CW, Bush AI, Mackinnon A, Macfarlane S, Mastwyk M, MacGregor L, Kiers L, Cherny RA, Li QX, Tammer A, Carrington D, Mavros C, Volitakis I, Xilinas M, Ames D, Davis S, Beyreuther K, Tanzi RE, Masters CL (2003) Metal-protein attenuation with iodochlorhydroxyquin (clioquinol) targeting A $\beta$ amyloid deposition and toxicity in Alzheimer's disease: a pilot phase 2 clinical trial. Arch Neurol 60, 1685-1691.

[13] Squitti R, Pasqualetti P, Dal Forno G, Moffa F, Cassetta E, Lupoi D, Vernieri F, Rossi L, Baldassini M, Rossini PM (2005) Excess of serum copper not related to ceruloplasmin in Alzheimer disease. Neurology 64, 1040-1046.

[14] Mattsson N, Zetterberg H, Hansson O, Andreasen N, Parnetti L, Jonsson M, Herukka SK, van der Flier WM, Blankenstein MA, Ewers M, Rich K, Kaiser E, Verbeek M, Tsolaki M, Mulugeta E, Rosen E, Aarsland D, Visser PJ, Schroder J, Marcusson J, de Leon M, Hampel H, Scheltens P, Pirttila T, Wallin A, Jonhagen ME, Minthon L, Winblad B, Blennow K (2009) CSF biomarkers and incipient Alzheimer disease in patients with mild cognitive impairment. JAMA 302, 385-393.

[15] Aisen PS, Gauthier S, Vellas B, Briand R, Saumier D, Laurin J, Garceau D (2007) Alzhemed: a potential treatment for Alzheimer's disease. Curr Alzheimer Res 4, 473-478.

[16] Salloway S, Sperling R, Gilman S, Fox NC, Blennow K, Raskind M, Sabbagh M, Honig LS, Doody R, van Dyck CH, Mulnard R, Barakos J, Gregg KM, Liu E, Lieberburg I, Schenk D, Black R, Grundman M (2009) A phase 2 multiple ascending dose trial of bapineuzumab in mild to moderate Alzheimer disease. Neurology 73, 2061-2070.

[17] Green RC, Schneider LS, Amato DA, Beelen AP, Wilcock G, Swabb EA, Zavitz KH, for the Tarenflurbil Phase 3 Study Group (2009) Effect of tarenflurbil on cognitive decline and activities of daily living in patients with mild Alzheimer disease: a randomized controlled trial. JAMA 302, 2557-2564.

[18] Harrison J, Minassian SL, Jenkins L, Black RS, Koller M, Grundman M (2007) A neuropsychological test battery for use in Alzheimer disease clinical trials. Arch Neurol 64, 13231329.

[19] Black R, Harrison J, Li D, Yang L, Booth K, Barlas S (2009). A Comparison of the Neuropsychological Test Battery (NTB) and ADAS-Cog Based on Alzheimerfls Disease Clinical Trials. In: International Conference on Alzheimer' s Disease. Vienna, P4-089. 